\title{
Capture Compounds for the functional isolation of nucleotide interacting proteins
}

\author{
The innovative Capture Compound mass spectrometry (CCMS) technology allows the functional \\ reduction of biological sample complexity. Small synthetic molecules (Capture Compounds ${ }^{\mathrm{TM}}$ ) are used \\ to interrogate native and even transmembrane proteins. Using nucleotide derivates as bait molecules \\ allows discovery, isolation and profiling of interacting proteins from complex biological samples. \\ Nucleotide-derived Capture Compounds are available as ready-to-use caproKits ${ }^{\text {TM }}$ for proteomic research.
}

The proteome is a highly complex mixture of diverse proteins and peptides varying in concentrations and states. To understand the time-dependent interplay of different signaling pathways and the structure and function of all involved proteins is a challenging task. Post-translational protein modifications constitute an additional layer of complexity and are important factors in the development of certain diseases ${ }^{1}$.

caprotec $^{\mathrm{TM}}$ has developed an innovative technology to substantially reduce the complexity of biological samples through a selective and functional isolation of proteins via trifunctional small molecules called Capture Compounds. Capture Compounds target proteins through reversible affinity interactions. A covalent bond between Capture Compound and target protein is generated by photo-cross-linking using the dedicated caproBox ${ }^{\mathrm{TM}}$. Streptavidin-coated beads are used to isolate captured proteins for further analyses (for example, western blot or mass spectrometry). Because of their straightforward workflow, CCMS experiments can be performed within a working day.

The functional isolation of proteome subsets based on small molecule-protein interactions is an increasingly popular approach in functional proteomics ${ }^{2}$. Entire protein families may be profiled on the basis of their common interaction with a small molecule. This is enabled by novel multifunctional small-molecule probes.

Nucleotide derivates are involved in many aspects of cellular signal transduction in a wide range of organisms. They can act as second messengers (e.g., cAMP or cGMP) in processes such as differentiation and apoptosis or as molecular switches in signaling cascades (e.g., GDP). Dysfunction of these signal cascades may result in the development of certain kinds of cancer, cardiovascular disorders or other diseases. The CCMS technology provides a distinctive hypothesis-generating approach toward a better understanding of the

\section{Erik Dülsner, Christian Jurinke \& Hubert Köster}

caprotec bioanalytics $\mathrm{GmbH}$, Berlin, Germany

Correspondence should be addressed to E.D. (erik.duelsner@caprotec.com)

orC.J. (christian.jurinke@caprotec.com). dependencies within different signaling pathways through identification and characterization of interacting partners via small molecules.

cAMP and cGMP (cyclic 3',5'-adenosine and guanosine monophosphates, respectively) are important second-messenger molecules ${ }^{3}$. The identification and the profiling of cyclic nucleotide monophosphate (cNMP)-binding proteins is vital to understanding the molecular basis of signaling events. In mammalian cells, a main interacting partner of CAMP is protein kinase A (PKA) which is conjugated to associated proteins, so-called A-kinase anchoring proteins (AKAPs). The active cAMP-PKA-AKAP complex phosphorylates downstream targets, and this results in an alteration of various signaling cascades affecting metabolism, gene transcription or apoptosis. More than 70 different AKAPs have been described, and a larger number is predicted. Figure 1 provides a classification of proteins isolated from HepG2 cell lysate using cAMP Capture Compound and shows the complexity of the cAMP interactome ${ }^{4}$.

A comparison of the capture profiles of CAMP- and cGMP-binding proteins in synaptosomes reveals that the CAMP caproKit robustly

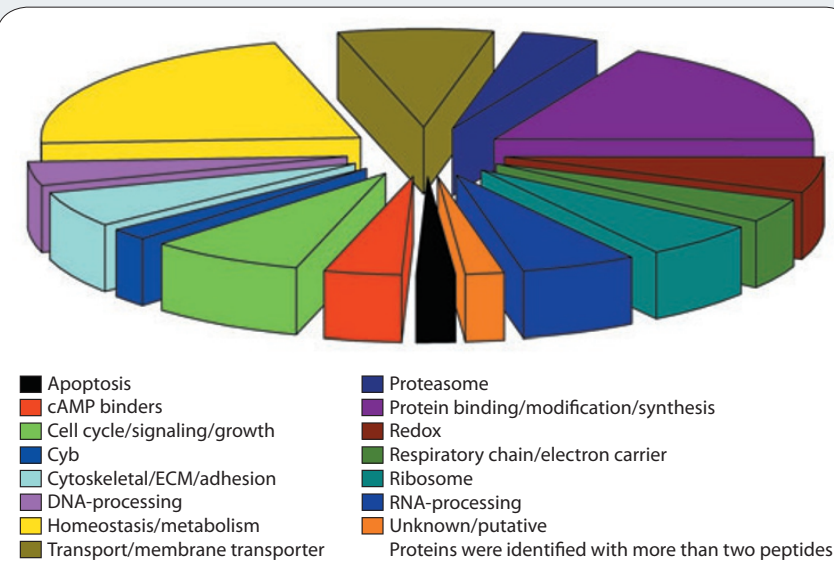

Figure 1 | Classification of captured proteins from HepG2 cell lysate using cAMP Capture Compounds, based on their biological process properties. Only proteins that were identified with more than two peptides are shown. 
Table 1 | GTPases captured from HEK 293 cells using the GDP Capture Compound

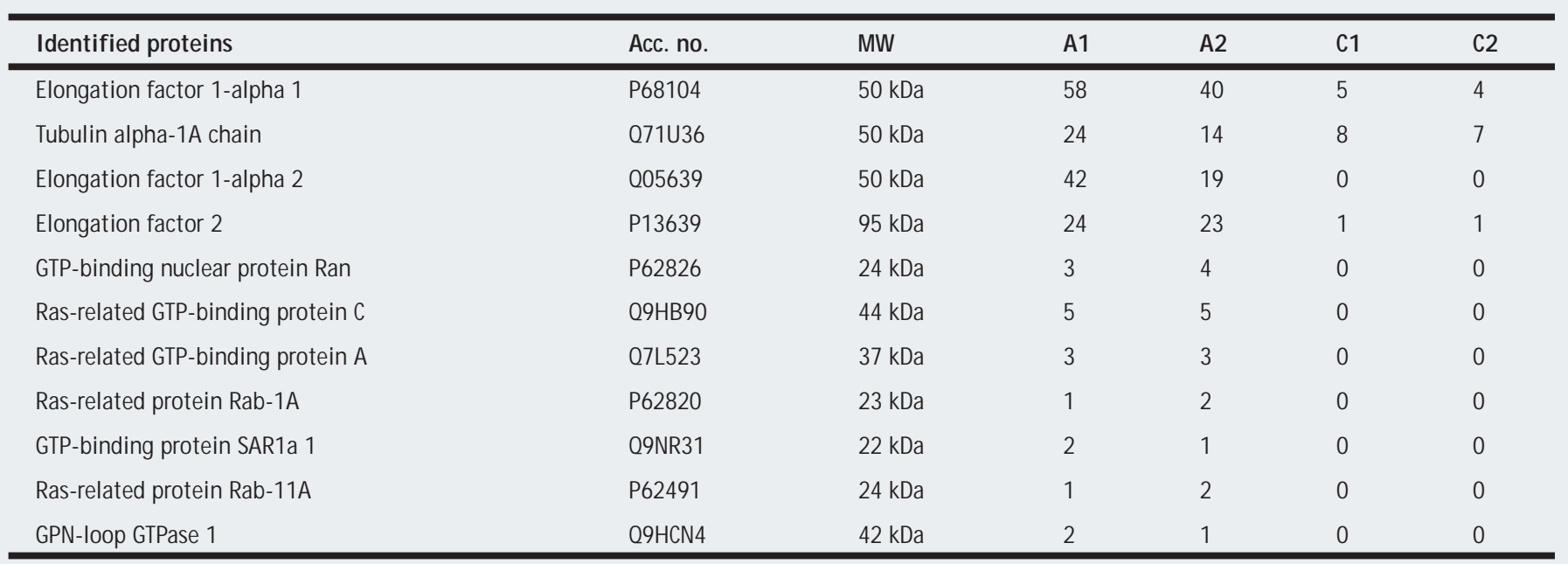

Two independent capture assays (A1, A2) compared to two competition controls (C1, C2) reveal the robust, selective capturing of GDP-binding proteins. The competitor is the free, non-bound selectivity function (in this case GDP). In the presence of a moderate excess of free competitor, the protein-Capture Compound interaction is suppressed. The captured proteins were OnBead-trypsinized and the peptide mixtures subjected to LC-MS. Spectral counts are given. MW, molecular weight. (Detailed results are provided in ref. 5.)

isolates CAMP-dependent protein kinases and some associated AKAP proteins (Fig. 2). Remarkably, the cAMP caproKit also permits the isolation of hyperpolarization-activated cyclic nucleotide-gated ( $\mathrm{HCN})$ ion channels at the endogenous protein level from very small amounts of synaptosome protein preparations. This has, to our knowledge, not been achieved in CAMP pull-down approaches reported in the literature. Because of the strong structural analogies between the cNMPs, the cGMP Capture Compound also captures some cAMPinteracting proteins, but with a much lower efficiency compared to the cAMP Capture Compound. For example, the phosphodiesterase PDE2A is known to be specifically stimulated by cGMP through binding to a cyclic nucleotide binding domain ${ }^{3}$. Only the cGMP Capture Compound isolates PDE2A, whereas the cAMP-binding protein Epac4 is captured exclusively by the cAMP Capture Compound (Fig. 2). Taken together, the CAMP and the cGMP caproKits can be effectively used for the isolation of cNMP-binding proteins from crude protein mixtures, with significantly distinct selectivity profiles for the respective Capture Compounds.

Guanosine nucleotide binding proteins (G proteins) are involved in second-messenger cascades and function as molecular switches. G proteins can be separated into two families: the heterotrimeric G proteins, which are activated by $\mathrm{G}$ protein-coupled receptors (GPCRs)

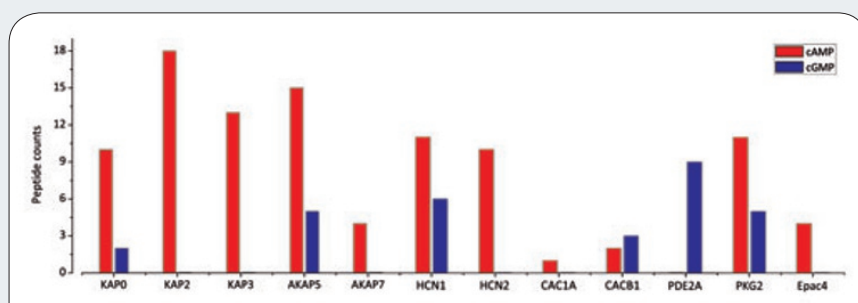

Figure $\mathbf{2}$ | Peptide counts of identified proteins from rat-brain preparations using CAMP (red) and cGMP (blue) caproKits, respectively. and consist of alpha, beta, and gamma subunits, and the monomeric small G proteins, which belong to the Ras superfamily of small GTPases. These proteins are homologous to the alpha subunit of heterotrimeric G proteins. Nearly 350 GPCRs are encoded by the human genome. Among them, roughly 150 have unknown functions.

Capturing in HEK 293 cells resulted in the specific enrichment of members of the Ras-like family of small GTPases (Table 1). In addition, several other GTPases, such as elongation factors 1 and 2 or Ran, were identified. These results demonstrate that the GDP Capture Compound robustly captures a wide range of different GTPases and therefore is a valuable tool for the proteomic profiling of this important protein family.

In conclusion, nucleotide derivate caproKits permit the isolation of important proteins, even transmembrane proteins, that are involved in many relevant signaling cascades. The CCMS technology helps to unravel small molecule-protein interactions with a higher efficiency than pull-down experiments.

1. Gygi, S.P., Rochon, Y., Franza, B.R. \& Aebersold, R. Correlation between protein and mRNA abundance in yeast. Mol. Cell. Biol. 19, 1720-1730 (1999).

2. Barglow, K.T. \& Cravatt, B.F. Activity-based protein profiling for the functional annotation of enzymes. Nat. Methods 4, 822-827 (2007).

3. Beavo, J.A. \& Brunton, L.L. Cyclic nucleotide research—still expanding after half a century. Nat. Rev. Mol. Cell Biol. 3, 710-718 (2002).

4. Luo, Y. et al. The CAMP capture compound mass spectrometry as a novel tool for targeting CAMP-binding proteins: from protein kinase A to potassium/ sodium hyperpolarization-activated cyclic nucleotide-gated channels. Mol. Cell. Proteomics 8, 2843-2856 (2009).

5. Luo, Y. et al. GDP-capture compound-a novel tool for the profiling of GTPases in pro- and eukaryotes by capture compound mass spectrometry (CCMS). J. Proteomics 73, 815-819 (2010).

This article was submitted to Nature Methods by a commercial organization and has not been peer reviewed. Nature Methods takes no responsibility for the accuracy or otherwise of the information provided. 